\title{
A new meaning and role of the notion of coherent prevision of multiple random quantities
}

\author{
Pierpaolo Angelini \\ Università "La Sapienza" di Roma \\ Dipartimento di Scienze Statistiche \\ pier.angelini@uniroma1.it
}

\begin{abstract}
I accept the principles of the theory of concordance into the domain of subjective probability in order to construct an original, geometric and well-organized theory of multiple random quantities. This acceptance is well-founded because the definition of concordance is implicit as well as the one of prevision of a random quantity and in particular of probability of an event. I limit myself to considering no more than two random quantities in this paper. A coherent prevision of a bivariate random quantity coincides with the notion of $\alpha$-product. Metric properties of the notion of $\alpha$-product mathematically characterize the notion of coherent prevision of a bivariate random quantity.
\end{abstract}

\section{Introduction}

Each random quantity justifies itself "a priori". Every finite partition of incompatible and exhaustive events representing a random quantity shows the possible ways in which a certain reality may be expressed. A multiplicity of possible values for every random quantity is only a formal construction that precedes the empirical observation by means of which a single value is realized among the ones of the set of the possible alternatives ([11]). Each event is a specific random quantity because it admits only two possible values. It does not admit more than two possible values like a random quantity. The same symbol $\mathbf{P}$ denotes both prevision of a random quantity and probability of an event ([14]). An event is conceptually a mental separation between sensations: it is actually a statement such that, by betting on it, we can establish in an unmistakable fashion whether it is true or false, that is to say, whether it has occurred or not and so whether the bet has been won or lost ([5]). Random quantities are studied by the logic of certainty as well as by the logic of the probable. It is methodologically fundamental to distinguish the logic of certainty from the logic of the probable ([6]). The logic of certainty does not use the notion of probability ([8]). It is called so for this reason. What is objectively possible belongs to the logic of certainty and it is different from what is subjectively probable. It makes sense to express one's subjective and

Keywords: vector homography, translation, affine tensor, antisymmetric tensor, $\alpha$-product, $\alpha$-norm 
non-predetermined opinion in terms of probability only in respect of what is possible or uncertain at a given instant. One always means uncertainty as a simple ignorance: it ceases only when one receives certain information. The logic of certainty is always characterized by two different and extreme aspects. The first aspect is negative because it deals with situations of non-knowledge or ignorance or uncertainty from which one determines the set of the possible alternatives of a random quantity: when a given numerical value is not either certain or impossible it is possible and it consequently belongs to such a set. The second aspect is positive because it deals with the definitive certainty expressed in the form of what is certainly true or certainly false. Every possible numerical value of a random quantity definitively becomes 0 or 1 when an empirical observation, referring to it, is made. Therefore, into the logic of certainty exist certain and impossible and possible regarding to the first aspect, true $=1$ and false $=0$ as final answers regarding to the second aspect. Hence, it is possible to observe that every set of the possible numerical values of each random quantity definitively becomes a smaller numerical set which is a Boolean algebra whose two elements are two idempotent numbers, 0 and 1 . Conversely, the notion of probability is of interest to an intermediate aspect which is included between the two extreme aspects characterizing the logic of certainty ([7], [9]). Indeed, the probability is distributed as a mass by a given individual over the domain of the possible alternatives before knowing which is the true alternative to be verified. This aspect is positive but it is weak and temporary because he is awaiting information which would give him the definitive certainty. In any case, what is logical is exact but it says nothing, so one has to consider the importance of what is extralogical: probability is exactly an extralogical notion in the sense that it is outside of the logic of certainty ([12], [13]). The value of the notion of probability does not transcend the psychological value that such a notion has with regard to each individual. Moreover, the value of the notion of probability is not independent of such a psychological value. Therefore, a living, elastic and psychological logic is considered: it is exactly the logic of the probable. Probability calculus has a very special character in this conceptual context because common sense plays the most essential role and it is analytically expressed as objective conditions of coherence ([15]).

\section{Univariate random quantities and their geometric rep- resentation}

A univariate quantity $X$ is really random for a given individual when he does not know its true numerical value. Therefore, he is in doubt between two or more than two possible values. These values belong to the set $I(X)=\left\{x^{1}, \ldots, x^{m}\right\}$. Only one possible numerical value of $I(X)$ will occur "a posteriori". It is possible to denote by ${ }_{(1)} S$ a set of univariate random quantities. Every random quantity belonging to the set ${ }_{(1)} S$ can be represented by a vector $\mathbf{x} \in E_{m}$, where $E_{m}$ is a vector space $m$-dimensional over the field $\mathbb{R}$ of real numbers having a Euclidean structure. The different possible values of every random quantity of ${ }_{(1)} S$ are $m$ in number. It turns out to be ${ }_{(1)} S \subseteq E_{m}$. The different possible values of $X$ belonging to the set $I(X)$ coincide with the different components of $\mathbf{x}$ and they can indifferently be denoted by a covariant or contravariant notation after choosing an orthonormal basis of $E_{m}$. I should exactly speak of components of $\mathbf{x}$ having upper indices or lower indices because I deal with an orthonormal basis of $E_{m}$. Indeed, the usage of the terms covariant and contravariant is geometrically meaningless because the covariant components of $\mathbf{x}$ coincide with the contravariant ones. Neverthe- 
less, it is appropriate the usage of this notation referring to them because a specific meaning regarding to them will be introduced. Having said that I will continue to use these terms. Thus, I choose a contravariant notation with respect to the components of $\mathbf{x}$ so it is possible to write $\mathbf{x}=\left(x^{i}\right)$ while I choose a covariant notation with respect to the components of $\mathbf{p}$ so it is possible to write $\mathbf{p}=\left(p_{i}\right)$, where $p_{i}$ represents a subjective probability assigned to $x^{i}, i=1, \ldots, m$, by a given individual. Hence, different individuals whose state of knowledge is hypothetically identical may choose different $p_{i}$ because each of them may subjectively give greater attention to certain circumstances than to others ([10]). A given individual is into the domain of the logic of certainty when he considers only $\mathbf{x} \in E_{m}$ while he is into the domain of the logic of the probable when he considers an ordered pair of vectors. It is expressed by $(\mathbf{x}, \mathbf{p}) \subseteq E_{m}$. Thus, a prevision of $X$ is given by

$$
\mathbf{P}(X)=\bar{X}=x^{i} p_{i},
$$

where I imply the Einstein summation convention. This prevision is coherent when one has $0 \leq p_{i} \leq 1, i=1, \ldots, m$, as well as $\sum_{i=1}^{m} p_{i}=1$ ([1]). This implies that a coherent prevision of $X$ always satisfies the inequality inf $I(X) \leq \mathbf{P}(X) \leq \sup I(X)$ and it is also linear, that is to say, one has $\mathbf{P}(a X+b Y+c Z+\ldots)=a \mathbf{P}(X)+b \mathbf{P}(Y)+c \mathbf{P}(Z)+\ldots$ for any finite number of univariate random quantities, with $a, b, c, \ldots$ any real numbers. In particular, from $\mathbf{P}(X+Y)=\mathbf{P}(X)+\mathbf{P}(Y)$ follows an additivity property of $\mathbf{P}$. A coherent prevision of $X$ can be expressed by means of the vector $\overline{\mathbf{x}}=\left(\bar{x}^{i}\right)$ that allows to define the transformed random quantity ${ }_{X} t$ : it is represented by the vector ${ }_{\mathbf{x}} \mathbf{t}=\mathbf{x}-\overline{\mathbf{x}}$ whose contravariant components are given by

$$
\mathrm{x}^{i}=x^{i}-\bar{x}^{i}
$$

This linear transformation of $X$ is a change of origin. A coherent prevision of the transformed random quantity ${ }_{X} t$ is necessarily given by

$$
\mathbf{P}\left({ }_{X} t\right)=\left(x^{i}-\bar{x}^{i}\right) p_{i}=0 .
$$

The $\alpha$-norm of the vector $\mathbf{x}$ is expressed by

$$
\|\mathbf{x}\|_{\alpha}^{2}=\left(x^{i}\right)^{2} p_{i} .
$$

It is the square of the quadratic mean of $X$. It turns out to be $\|\mathbf{x}\|_{\alpha}^{2} \geq 0$. In particular, one writes $\|\mathbf{x}\|_{\alpha}^{2}=0$ when the possible values of $X$ are all null: this is a degenerate case. Hence, one says that the $\alpha$-norm of the vector $\mathbf{x}$ is strictly positive. The $\alpha$-norm of the vector representing ${ }_{X} t$ is given by

$$
\left\|_{\mathbf{X}} \mathbf{t}\right\|_{\alpha}^{2}=\left({ }_{\mathbf{x}} t^{i}\right)^{2} p_{i}=\sigma_{X}^{2} .
$$

It represents the variance of $X$ in a vectorial fashion. I will later explain why I use the term $\alpha$-norm.

\section{Bivariate random quantities and their geometric rep- resentation}

It is possible to denote by ${ }_{(2)} S^{(2)}$ a set of bivariate random quantities and by $X_{12} \equiv$ $\left\{{ }_{1} X,{ }_{2} X\right\}$ a generic bivariate random quantity of this set. A pair of univariate random 
quantities $\left({ }_{1} X,{ }_{2} X\right)$ evidently represents an ordered pair of univariate random quantities which are the components of $X_{12}$. Each element of ${ }_{(2)} S^{(2)}$ can be represented by an affine tensor of order 2 denoted by $T \in E_{m}^{(2)}=E_{m} \otimes E_{m}$. Therefore, the possible values of $X_{12}$ coincide with the numerical values of the components of $T$. The dimension of $E_{m}$ as well as the number of the different possible values of every univariate random quantity of $X_{12}$ is expressed by $m$. Thus, $T$ is an element of a vector space $m^{2}$-dimensional. Moreover, it turns out to be ${ }_{(2)} S^{(2)} \subseteq E_{m}^{(2)}$. I choose an orthonormal basis of $E_{m}$ in order to represent the possible values of $X_{12}$. These values coincide with the contravariant components of $T$ so it is possible to write

$$
T={ }_{(1)} \mathbf{x} \otimes{ }_{(2)} \mathbf{x}={ }_{(1)} x^{i_{1}}{ }_{(2)} x^{i_{2}} \mathbf{e}_{i_{1}} \otimes \mathbf{e}_{i_{2}} .
$$

The tensor representation of $X_{12}$ expressed by (6) depends on $\left({ }_{1} X,{ }_{2} X\right)$. Indeed, if one considers a different ordered pair $\left({ }_{2} X,{ }_{1} X\right)$ of univariate random quantities one obtains a different tensor representation of $X_{12}$ expressed by

$$
T={ }_{(2)} \mathbf{x} \otimes{ }_{(1)} \mathbf{x}={ }_{(2)} x^{i_{2}}{ }_{(1)} x^{i_{1}} \mathbf{e}_{i_{2}} \otimes \mathbf{e}_{i_{1}}
$$

because the tensor product is not commutative ([22]). Therefore, the components of $T$ expressed by (7) are not the same of the ones expressed by (6). Both these formulas express an affine tensor of order 2 whose components are different. I could consider two vectors of $E_{3}$

$$
{ }_{(1)} \mathbf{x}={ }_{(1)} x^{1} \mathbf{e}_{1}+{ }_{(1)} x^{2} \mathbf{e}_{2}+{ }_{(1)} x^{3} \mathbf{e}_{3}
$$

and

$$
{ }_{(2)} \mathbf{x}={ }_{(2)} x^{1} \mathbf{e}_{1}+{ }_{(2)} x^{2} \mathbf{e}_{2}+{ }_{(2)} x^{3} \mathbf{e}_{3}
$$

in order to realize that it turns out to be ${ }_{(1)} \mathbf{x} \otimes_{(2)} \mathbf{x} \neq_{(2)} \mathbf{x} \otimes_{(1)} \mathbf{x}$ by summing over all the values of the indices. Then, I must consider (6) and (7) at the same time in order to release a tensor representation of $X_{12}$ from any ordered pair of univariate random quantities which can be considered, $\left({ }_{1} X,{ }_{2} X\right)$ or $\left({ }_{2} X,{ }_{1} X\right)$. In fact, when $m=3$ and I express $T$ by means of (6) and (7) I observe that three of nine summands are equal, so it is possible to say that the possible values of a bivariate random quantity must be expressed by the components of an antisymmetric tensor of order 2 . It is expressed by

$$
T=\sum_{i_{1}<i_{2}}\left({ }_{(1)} x^{i_{1}}{ }_{(2)} x^{i_{2}}-{ }_{(1)} x^{i_{2}}{ }_{(2)} x^{i_{1}}\right) \mathbf{e}_{i_{1}} \otimes \mathbf{e}_{i_{2}} .
$$

The number of the components of an antisymmetric tensor of order 2 is evidently different from the one of the components of an affine tensor of the same order. Thus, a tensor representation based on an antisymmetric tensor of order 2 does not depend either on $\left({ }_{1} X,{ }_{2} X\right)$ or $\left({ }_{2} X,{ }_{1} X\right)$. I choose it in order to represent a generic bivariate random quantity $X_{12}$. Therefore, ${ }_{12} f$ is an antisymmetric tensor of order 2 called the tensor of the possible values of $X_{12}$. The contravariant components of ${ }_{12} f$ expressed by

$$
{ }_{12} f^{\left(i_{1} i_{2}\right)}=\left|\begin{array}{ll}
(1)^{x^{i_{1}}} & (1)^{x_{2}} \\
(2)^{x^{i_{1}}} & (2)^{x_{2}}
\end{array}\right|
$$

represent the possible values of $X_{12}$ in a tensorial fashion. These components are equal to 0 when they have equal indices. It is evident that the vector space of the antisymmetric tensors of order 2 does not have a dimension equal to $m^{2}$ but it has a dimension 
equal to $\left(\begin{array}{c}m \\ 2\end{array}\right)$. Now, I must introduce the probabilities into this geometric representation of $X_{12}$. It is possible to say that the tensor of the joint probabilities $p=\left(p_{i_{1} i_{2}}\right)$ is an affine tensor of order 2 whose covariant components represent those probabilities related to the ordered pairs of components of vectors representing the marginal univariate random quantities ${ }_{1} X$ and ${ }_{2} X$ of $X_{12}$. Then, a coherent prevision of $X_{12}$ is expressed by

$$
\mathbf{P}\left(X_{12}\right)=\bar{X}_{12}={ }_{(1)} x^{i_{1}}{ }_{(2)} x^{i_{2}} p_{i_{1} i_{2}},
$$

so it is also possible to consider the affine tensor of order 2 denoted by ${ }_{12} \bar{x}$ whose contravariant components are expressed by ${ }_{12} \bar{x}^{i_{1} i_{2}}$. They are all equal. In order to define the covariant components of ${ }_{12} f$ I must consider those vector homographies that allow me to pass from the contravariant components of a type of vector to the covariant components of another type of vector by means of the tensor of the joint probabilities under consideration. Indeed, the covariant components of ${ }_{12} f$ represent those probabilities related to the possible values of each marginal univariate random quantity of $X_{12}$. These components are obtained by summing the probabilities related to the ordered pairs of components of ${ }_{(1)} \mathbf{x}$ and ${ }_{(2)} \mathbf{x}$ : putting the joint probabilities into a two-way table I consider the totals of each row and the totals of each column of the table as covariant components of ${ }_{12} f$. In analytic terms one has ${ }_{(1)} x^{i_{1}} p_{i_{1} i_{2}}={ }_{(1)} x_{i_{2}}$ and ${ }_{(2)} x^{i_{2}} p_{i_{1} i_{2}}={ }_{(2)} x_{i_{1}}$ by virtue of a specific convention that I introduce: when the covariant indices to right-hand side vary over all their possible values I obtain two sequences of values representing those probabilities related to the possible values of each marginal univariate random quantity of $X_{12}$. They are the covariant components of ${ }_{12} f$. It turns out to be

$$
{ }_{12} f_{\left(i_{1} i_{2}\right)}=\left|\begin{array}{cc}
{ }_{(1)} x_{i_{1}} & (1)^{x} x_{i_{2}} \\
{ }_{(2)} x_{i_{1}} & (2)^{x} x_{i_{2}}
\end{array}\right|=\left|\begin{array}{ll}
{ }_{(1)} x^{i_{2}} p_{i_{2} i_{1}} & (1)^{x^{i_{1}}} p_{i_{1} i_{2}} \\
(2)^{x^{i_{2}}} p_{i_{2} i_{1}} & (2)^{x^{i_{1}}} p_{i_{1} i_{2}}
\end{array}\right| .
$$

The covariant indices of the tensor $p$ can be interchanged when it is necessary so one has, for instance, ${ }_{(1)} x^{i_{1}} p_{i_{1} i_{2}}={ }_{(1)} x^{i_{1}} p_{i_{2} i_{1}}$. Each ordered pair of vectors $\left(_{(1)} \mathbf{x},{ }_{(2)} \mathbf{x}\right)$ mathematically determines an affine tensor of order 2 when a given individual is into the subjective domain of the logic of the probable. Each ordered pair of vectors $\left(_{(1)} \mathbf{x},{ }_{(2)} \mathbf{x}\right)$ represents two univariate random quantities, ${ }_{1} X$ and ${ }_{2} X$, into $E_{m}$ ([23]). Both these univariate random quantities belong to the set denoted by ${ }_{(2)} S^{(1)}$ so it turns out to be ${ }_{(2)} S^{(1)} \subseteq E_{m}$. On the other hand it is possible to write ${ }_{(2)} S^{(1)} \otimes{ }_{(2)} S^{(1)}={ }_{(2)} S^{(2)}$, so I reach the vector space of the antisymmetric tensors of order 2 by anti-symmetrization. It is denoted by ${ }_{(2)} S^{(2) \wedge}$. One has evidently ${ }_{(2)} S^{(2) \wedge} \subseteq E_{m}^{(2) \wedge}$. I will show that a metric defined on ${ }_{(2)} S^{(2) \wedge}$ is the consequence of a metric defined on ${ }_{(2)} S^{(1)}$.

\section{Metric aspects related to univariate random quanti- ties}

The vector space of univariate random quantities which are the components of bivariate random quantities is denoted by ${ }_{(2)} S^{(1)} \subseteq E_{m}$. These univariate random quantities are represented by two vectors, ${ }_{(1)} \mathbf{x}$ and ${ }_{(2)} \mathbf{x}$, belonging to $E_{m}$. I deal with two ordered $m$ tuples of real numbers when I am into the objective domain of the possible alternatives. An affine tensor $p$ of order 2 must be added to the two vectors under consideration when I pass from the domain of the possible alternatives to the one of the evaluation of probabilities. Therefore, I always consider a triple of elements. I transform the vector 
${ }_{(2)} \mathbf{x}$ into the vector ${ }_{(2)} \mathbf{x}^{\prime}$ by means of the tensor $p$. Hence, it is possible to write the following dot product

$$
{ }_{(1)} \mathbf{x} \cdot{ }_{(2)} \mathbf{x}^{\prime}={ }_{(1)} x^{i_{1}}{ }_{(2)} x^{i_{2}} p_{i_{1} i_{2}}={ }_{(1)} x^{i_{1}}{ }_{(2)} x_{i_{1}}
$$

I note that

$$
{ }_{(2)} x_{i_{1}}={ }_{(2)} x^{i_{2}} p_{i_{1} i_{2}}={ }_{(2)} \mathbf{x}^{\prime}
$$

is a vector homography whose expressions are obtained by applying the Einstein summation convention. Then, the $\alpha$-product of two vectors, ${ }_{(1)} \mathbf{x}$ and ${ }_{(2)} \mathbf{x}$, is defined as a dot product of two vectors, ${ }_{(1)} \mathbf{x}$ and ${ }_{(2)} \mathbf{x}^{\prime}$, so I write

$$
{ }_{(1)} \mathbf{X} \odot_{(2)} \mathbf{x}={ }_{(1)} \mathbf{x} \cdot{ }_{(2)} \mathbf{x}^{\prime} .
$$

In particular, the $\alpha$-norm of the vector ${ }_{(1)} \mathbf{x}$ is given by

$$
\left\|_{(1)} \mathbf{x}\right\|_{\alpha}^{2}={ }_{(1)} x^{i_{1}}{ }_{(1)} x^{i_{1}} p_{i_{1} i_{1}}={ }_{(1)} x^{i_{1}}{ }_{(1)} x_{i_{1}} .
$$

Now, I can explain why I use this term: I use it because I refer to the $\alpha$-criterion of concordance introduced by Gini ([24], [25]). There actually exist different criteria of concordance shown by Gini in addition to the $\alpha$-criterion. Nevertheless, by considering quadratic measures of concordance it always suffices to use the $\alpha$-criterion ([?]). When I pass from the notion of $\alpha$-product to the one of $\alpha$-norm I say that the corresponding possible values of the two univariate random quantities under consideration are equal. I also say that the corresponding probabilities are equal. Therefore, the covariant components of the tensor $p=\left(p_{i_{1} i_{2}}\right)$ having different numerical values as indices are null. Thus, I say that the absolute maximum of concordance is realized. Given the vector $\mathbf{y}={ }_{(1)} \mathbf{x}+\lambda_{(2)} \mathbf{x}$, its $\alpha$-norm is expressed by

$$
\|\mathbf{y}\|_{\alpha}^{2}=\left\|_{(1)} \mathbf{x}\right\|_{\alpha}^{2}+2 \lambda\left(_{(1)} \mathbf{x} \odot{ }_{(2)} \mathbf{x}\right)+\lambda^{2}\left\|_{(2)} \mathbf{x}\right\|_{\alpha}^{2} .
$$

It is always possible to write $\|\mathbf{y}\|_{\alpha}^{2} \geq 0$. Moreover, the right-hand side of (16) is a quadratic trinomial whose variable is $\lambda \in \mathbb{R}$, so I must consider a quadratic inequation. All real numbers fulfill the condition stated in the form $\|\mathbf{y}\|_{\alpha}^{2} \geq 0$. This means that the discriminant of the associated quadratic equation is non-positive. I write

$$
\Delta_{\lambda}=4\left[\left(_{(1)} \mathbf{x} \odot_{(2)} \mathbf{x}\right)^{2}-\left\|_{(1)} \mathbf{x}\right\|_{\alpha}^{2}\left\|_{(2)} \mathbf{x}\right\|_{\alpha}^{2}\right] .
$$

Given $\Delta_{\lambda} \leq 0$, it turns out to be

$$
\left({ }_{(1)} \mathbf{x} \odot{ }_{(2)} \mathbf{x}\right)^{2} \leq\left\|_{(1)} \mathbf{x}\right\|_{\alpha}^{2}\left\|_{(2)} \mathbf{x}\right\|_{\alpha}^{2},
$$

so I obtain

$$
\left.\right|_{(1)} \mathbf{x} \odot{ }_{(2)} \mathbf{x} \mid \leq\left\|_{(1)} \mathbf{x}\right\|_{\alpha}\left\|_{(2)} \mathbf{x}\right\|_{\alpha}
$$

The expression (17) is called the Schwarz's $\alpha$-generalized inequality. When $\lambda=1$ one has $\mathbf{y}={ }_{(1)} \mathbf{x}+{ }_{(2)} \mathbf{x}$. By replacing $\left(_{(1)} \mathbf{x} \odot{ }_{(2)} \mathbf{x}\right)$ into (16) with $\left\|_{(1)} \mathbf{x}\right\|_{\alpha}\left\|_{(2)} \mathbf{x}\right\|_{\alpha}$ one has the square of a binomial given by

$$
\left\|_{(1)} \mathbf{x}+{ }_{(2)} \mathbf{x}\right\|_{\alpha}^{2}=\left\|_{(1)} \mathbf{x}\right\|_{\alpha}^{2}+2\left\|_{(1)} \mathbf{x}\right\|_{\alpha}\left\|_{(2)} \mathbf{x}\right\|_{\alpha}+\left\|_{(2)} \mathbf{x}\right\|_{\alpha}^{2},
$$

so one obtains

$$
\left\|_{(1)} \mathbf{x}+{ }_{(2)} \mathbf{x}\right\|_{\alpha} \leq\left\|_{(1)} \mathbf{x}\right\|_{\alpha}+\left\|_{(2)} \mathbf{x}\right\|_{\alpha}
$$


The expression (18) is called the $\alpha$-triangle inequality. Dividing by $\left\|{ }_{(1)} \mathbf{x}\right\|_{\alpha}\left\|_{(2)} \mathbf{x}\right\|_{\alpha}$ both sides of (17) one has

$$
\left|\frac{{ }_{(1)} \mathbf{x} \odot{ }_{(2)} \mathbf{x}}{\left\|_{(1)} \mathbf{x}\right\|_{\alpha}\left\|_{(2)} \mathbf{x}\right\|_{\alpha}}\right| \leq 1
$$

that is to say,

$$
-1 \leq \frac{{ }_{11} \mathbf{x} \odot{ }_{(2)} \mathbf{x}}{\|\|_{(1)} \mathbf{x}\left\|_{\alpha}\right\|_{(2)} \mathbf{x} \|_{\alpha}} \leq 1,
$$

so there exists a unique angle $\gamma$ such that $0 \leq \gamma \leq \pi$ and such that

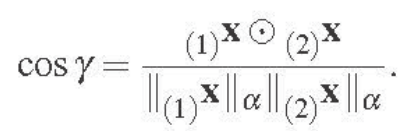

It is possible to define this angle to be the angle between the two vectors ${ }_{(1)} \mathbf{x}$ and (2) $\mathbf{x}$. By considering the expression (14) it is also possible to define it to be the angle between ${ }_{(1)} \mathbf{x}$ and ${ }_{(2)} \mathbf{x}^{\prime}$. The two vectors ${ }_{(1)} \mathbf{t}$ and ${ }_{(2)} \mathbf{t}$ represent the two transformed random quantities ${ }_{1} X^{t}$ and ${ }_{2} X^{t}$ defined on ${ }_{1} X$ and ${ }_{2} X$. Then, their $\alpha$-product is given by

$$
{ }_{(1)} \mathbf{t} \odot{ }_{(2)} \mathbf{t}={ }_{(1)} t^{i_{1}}{ }_{(2)} t_{i_{1}}={ }_{(1)} t^{i_{1}}{ }_{(2)} t^{i_{2}} p_{i_{2} i_{1}} .
$$

It represents the covariance of ${ }_{1} X$ and ${ }_{2} X$ in a vectorial fashion. When one considers the expression (19) referring to the vectors ${ }_{(1)} \mathbf{t}$ and ${ }_{(2)} \mathbf{t}$ it becomes

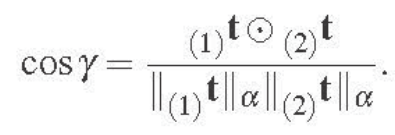

It expresses the Pearson $\alpha$-generalized correlation coefficient.

\section{Metric aspects related to bivariate random quantities}

I deal with the vector space denoted by ${ }_{(2)} S^{(2) \wedge}$ whose elements are antisymmetric tensors of order 2. Nevertheless, by introducing the notion of $\alpha$-product of two antisymmetric tensors of order 2 I must underline a very important point: it is not necessary to refer to the bivariate random quantity $X_{12}$ in order to introduce that antisymmetric tensor whose covariant components are represented like into the expression (11). Therefore, it is also possible to consider a bivariate random quantity denoted by $X_{34}$ as well as an antisymmetric tensor of order 2 denoted by ${ }_{34} f$ whose covariant components are expressed by

$$
{ }_{34} f_{\left(i_{1} i_{2}\right)}=\left|\begin{array}{ll}
(3)^{x} x_{i_{1}} & (3)^{x_{i_{2}}} \\
(4)^{x_{i_{1}}} & (4)^{x} x_{i_{2}}
\end{array}\right|=\left|\begin{array}{ll}
{ }_{(3)^{x_{2}}} p_{i_{2} i_{1}} & (3)^{x^{i_{1}}} p_{i_{1} i_{2}} \\
{ }_{(4)^{x_{2}}} p_{i_{2} i_{1}} & (4)^{x^{i_{1}}} p_{i_{1} i_{2}}
\end{array}\right| .
$$

Thus, it is possible to extend to the antisymmetric tensors ${ }_{12} f$ and ${ }_{34} f$ the notion of $\alpha$-product. This means that one can examine the domain of the possible alternatives in a more complete fashion ([16]). Then, one has

$$
{ }_{12} f^{\left(i_{1} i_{2}\right)} \odot{ }_{34} f_{\left(i_{1} i_{2}\right)}=\frac{1}{2}\left|\begin{array}{ll}
(1)^{x_{1}} & (1)^{x_{2}} \\
{ }_{(2)} x^{x_{1}} & (2)^{x_{2}}
\end{array}\right|\left|\begin{array}{cc}
(3)^{x_{i_{1}}} & (3)^{x_{i_{2}}} \\
(4)^{x_{i_{1}}} & (4)^{x_{i_{2}}}
\end{array}\right|,
$$


where it appears $\frac{1}{2}$ because one has always two permutations into the two determinants: one of these permutations is "good" when it turns out to be $i_{1}<i_{2}$ regarding to ${ }_{(1)} x^{i_{1}}{ }_{(2)} x^{i_{2}}$ and ${ }_{(3)} x_{i_{1}(4)} x_{i_{2}}$ while the other is "no good" because it turns out to be $i_{2}>i_{1}$ regarding to ${ }_{(1)} x^{i_{2}}{ }_{(2)} x^{i_{1}}$ and ${ }_{(3)} x_{i_{2}(4)} x_{i_{1}}$. Hence, I am in need of returning to normality by means of $\frac{1}{2}$. Such a normality is evidently represented by $i_{1}<i_{2}$. I need different affine tensors of order 2 in order to make a calculation given by the expression (23). These tensors of the joint probabilities allow me of defining the bivariate random quantities $X_{13}, X_{14}, X_{23}$ and $X_{24}$. Thus, one has

$$
{ }_{12} f \odot_{34} f=\left|\begin{array}{ll}
{ }_{(1)}{ }^{i_{1}}{ }_{(3)} x^{i_{2}} p_{i_{2} i_{1}}^{(13)} & (1)^{x_{2}}{ }_{(4)} x^{i_{1}} p_{i_{1} i_{2}}^{(14)} \\
{ }_{(2)} x^{x_{1}{ }_{(3)} x^{i_{2}} p_{i_{2} i_{1}}^{(23)}} & (2)^{x_{2}}{ }_{(4)} x^{i_{1}} p_{i_{1} i_{2}}^{(24)}
\end{array}\right|
$$

In particular, the $\alpha$-norm of the tensor ${ }_{12} f$ is given by

$$
\left\|_{12} f\right\|_{\alpha}^{2}={ }_{12} f \odot{ }_{12} f={ }_{12} f^{\left(i_{1} i_{2}\right)}{ }_{12} f_{\left(i_{1} i_{2}\right)}
$$

so it turns out to be

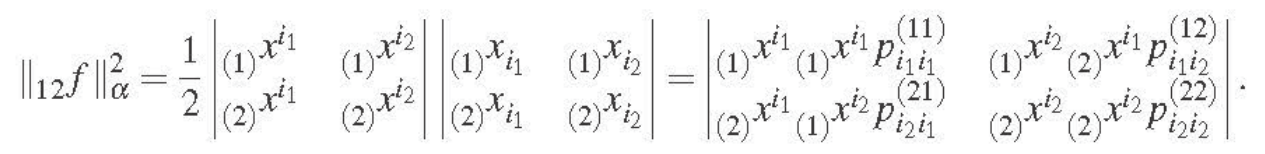

Anyway, it is always possible to write

$$
{ }_{12} f \odot_{34} f=\left|\begin{array}{ll}
{ }_{(1)} \mathbf{x} \odot{ }_{(3)} \mathbf{x} & { }_{(1)} \mathbf{x} \odot{ }_{(4)} \mathbf{x} \\
{ }_{(2)} \mathbf{x} \odot{ }_{(3)} \mathbf{x} & { }_{(2)} \mathbf{x} \odot{ }_{(4)} \mathbf{x}
\end{array}\right|
$$

as well as

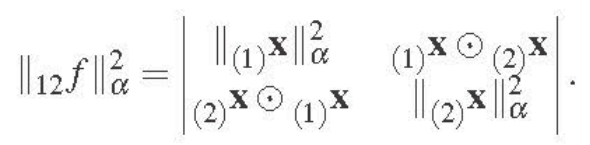

The $\alpha$-norm of the tensor ${ }_{12} f$ is again strictly positive. It is equal to 0 when the components of ${ }_{12} f$ are null. Nevertheless, this does not mean that the components of the two vectors founding the tensor are null. Indeed, it suffices that one writes ${ }_{(1)} \mathbf{x}=\lambda_{(2)} \mathbf{x}$, with $\lambda \in \mathbb{R}$, in order to obtain

$$
\left\|_{12} f_{\lambda}\right\|_{\alpha}^{2}=\frac{1}{2}\left|\begin{array}{cc}
\lambda_{(2)} x^{i_{1}} & \lambda_{(2)} x^{x_{2}} \\
x^{i_{1}} & { }_{(2)} x^{i_{2}}
\end{array}\right|\left|\begin{array}{cc}
\lambda_{(2)} x_{i_{1}} & \lambda_{(2)} x_{i_{2}} \\
(2)^{x_{i_{1}}} & \left(_{(2)} x_{i_{2}}\right.
\end{array}\right|=\left|\begin{array}{cc}
\lambda^{2}\left\|_{(2)} \mathbf{x}\right\|_{\alpha}^{2} & \lambda\left\|_{(2)} \mathbf{x}\right\|_{\alpha}^{2} \\
\lambda\left\|_{(2)} \mathbf{x}\right\|_{\alpha}^{2} & \left\|_{(2)} \mathbf{x}\right\|_{\alpha}^{2}
\end{array}\right|=0 .
$$

I define the tensor $f$ as a linear combination of ${ }_{12} f$ and ${ }_{34} f$ such that I can write $f={ }_{12} f+\lambda_{34} f$, with $\lambda \in \mathbb{R}$. Then, the Schwarz's $\alpha$-generalized inequality becomes

$$
\left|{ }_{12} f \odot{ }_{34} f\right| \leq\left\|_{12} f\right\|_{\alpha}\left\|_{34} f\right\|_{\alpha},
$$

the $\alpha$-triangle inequality becomes

$$
\left\|_{12} f+{ }_{34} f\right\|_{\alpha} \leq\left\|_{12} f\right\|_{\alpha}+\left\|_{34} f\right\|_{\alpha},
$$

while the cosine of the angle $\gamma$ becomes

$$
\cos \gamma=\frac{{ }_{12} f \odot_{34} f}{\left\|_{12} f\right\|_{\alpha}\left\|_{34} f\right\|_{\alpha}} .
$$


It is possible to consider two transformed univariate random quantities which are respectively ${ }_{1} X^{t}$ and ${ }_{2} X^{t}$. They are represented by the vectors ${ }_{(1)} \mathbf{t}$ and ${ }_{(2)} \mathbf{t}$ whose contravariant components are given by ${ }_{(1)} t^{i}={ }_{(1)} x^{i}-{ }_{(1)} \bar{x}^{i}$ and ${ }_{(2)} t^{i}={ }_{(2)} x^{i}-{ }_{(2)} \bar{x}^{i}$. Therefore, it is possible to introduce an antisymmetric tensor of order 2 denoted by ${ }_{12} t$ which characterizes the transformed bivariate random quantity denoted by $x_{12} t$. Then, the contravariant components of this tensor are given by

$$
{ }_{12} t^{\left(i_{1} i_{2}\right)}=\left|\begin{array}{ll}
(1)^{t_{1}} & (1)^{t_{2}} \\
(2)^{i_{1}} & (2)^{i_{2}}
\end{array}\right|
$$

Its covariant components are given by

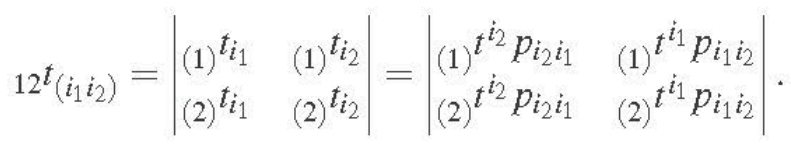

The $\alpha$-product of the two tensors ${ }_{12} t$ and ${ }_{34} t$ is given by

$$
{ }_{12} t \odot{ }_{34} t=\left|\begin{array}{ll}
{ }_{(1)} \mathbf{t} \odot{ }_{(3)} \mathbf{t} & { }_{(1)} \mathbf{t} \odot{ }_{(4)} \mathbf{t} \\
{ }_{(2)} \mathbf{t} \odot{ }_{(3)} \mathbf{t} & { }_{(2)} \mathbf{t} \odot{ }_{(4)} \mathbf{t}
\end{array}\right| .
$$

The $\alpha$-norm of the tensor ${ }_{12} t$ is given by

$$
\left\|_{12} t\right\|_{\alpha}^{2}=\left|\begin{array}{cc}
\left\|_{(1)} \mathbf{t}\right\|_{\alpha}^{2} & \left({ }_{(1)} \mathbf{t} \odot{ }_{(2)} \mathbf{t}\right. \\
{ }_{(2)} \odot_{(1)} \mathbf{t} & \left\|_{(2)} \mathbf{t}\right\|_{\alpha}^{2}
\end{array}\right| .
$$

The cosine of the angle $\gamma$ is given by

$$
\cos \gamma=\frac{12^{t} \odot{ }_{34} t}{\|12 t\|_{\alpha}\left\|_{34} t\right\|_{\alpha}} .
$$

It will be possible to realize completely in due time that all these expressions are at the base of meaningful quantitative relationships between multiple random quantities characterizing an original, well-organized and extensive theory that I want to introduce into the domain of subjective probability.

\section{Metric properties of the prevision of a generic bivari- ate random quantity}

The notion of $\alpha$-product depends on three elements which are two vectors of $E_{m},{ }_{(1)} \mathbf{x}$ and ${ }_{(2)} \mathbf{x}$, and one affine tensor $p=\left(p_{i_{1} i_{2}}\right)$ of order 2 belonging to $E_{m}^{(2)}=E_{m} \otimes E_{m}$. Given any ordered pair of vectors, $p$ is uniquely determined as a geometric object. This implies that each covariant component of $p$ is always a subjective probability intrinsically coherent ([4]). With regard to some problem that may be considered it is possible that all reasonable people share each covariant component of $p$. Nevertheless, an opinion in terms of probability shared by many people always remains a subjective opinion. It is meaningless to say that it is objectively exact. Indeed, a sum of many subjective opinions in terms of probability can never lead to an objectively correct conclusion ([3]). Thus, given a bivariate random quantity $X_{12} \equiv\left\{{ }_{1} X,{ }_{2} X\right\}$, its coherent 
prevision $\mathbf{P}\left(X_{12}\right)$ is an $\alpha$-product whose metric properties remain unchanged by extending them to $\mathbf{P}$. Therefore, $\mathbf{P}$ is an $\alpha$-commutative prevision because it is possible to write

$$
\mathbf{P}\left({ }_{1} X_{2} X\right)=\mathbf{P}\left({ }_{2} X_{1} X\right)
$$

$\mathbf{P}$ is an $\alpha$-associative prevision because it is possible to write

$$
\mathbf{P}\left[\left(\lambda_{1} X\right)_{2} X\right]=\mathbf{P}\left[{ }_{1} X\left(\lambda_{2} X\right)\right]=\lambda \mathbf{P}\left({ }_{1} X_{2} X\right), \forall \lambda \in \mathbb{R},
$$

$\mathbf{P}$ is an $\alpha$-distributive prevision because it is possible to write

$$
\mathbf{P}\left[\left({ }_{1} X+{ }_{2} X\right){ }_{3} X\right]=\mathbf{P}\left({ }_{1} X X_{3} X\right)+\mathbf{P}\left({ }_{2} X X_{3} X\right) .
$$

Moreover, when one writes

$$
\mathbf{P}\left({ }_{1} X_{2} X\right)=\mathbf{P}\left({ }_{2} X_{1} X\right)=0,
$$

and all possible values of ${ }_{1} X$ and ${ }_{2} X$ are not null, one says that ${ }_{1} X$ and ${ }_{2} X$ are $\alpha$ orthogonal univariate random quantities. In particular, one observes that the $\alpha$-distributive property of prevision implies that the covariant components of the affine tensor $p^{(13)}$ are equal to the ones of the affine tensor $p^{(23)}$. Moreover, the covariant components of the affine tensor related to the two univariate random quantities ${ }_{1} X+{ }_{2} X$ and ${ }_{3} X$ are the same of the ones of $p^{(13)}$ and $p^{(23)}$. By considering a bivariate random quantity one finally says that its prevision $\mathbf{P}$ is bilinear. If the possible values of the two univariate random quantities of $X_{12} \equiv\left\{{ }_{1} X,{ }_{2} X\right\}$ are correspondingly equal and the covariant components of the tensor $p=\left(p_{i_{1} i_{2}}\right)$ having different numerical values as indices are null, then $\mathbf{P}\left(X_{12}\right)=\mathbf{P}\left({ }_{1} X_{2} X\right)=\mathbf{P}\left({ }_{2} X_{1} X\right)$ coincides with the $\alpha$-norm of ${ }_{(1)} \mathbf{x}={ }_{(2)} \mathbf{x}$. If $\mathbf{P}\left(X_{12}\right)$ is a coherent prevision of $X_{12} \equiv\left\{{ }_{1} X,{ }_{2} X\right\}$, then its univariate random quantities, ${ }_{1} X$ and ${ }_{2} X$, represent two separate and finite partitions of incompatible and exhaustive events whose non-negative probabilities sum to 1 . These are objective conditions of coherence ([2], [19], [20]). It is evident that each covariant component of $p=\left(p_{i_{1} i_{2}}\right)$ represents a probability of the joint of two events which includes a conditional probability of an event given the other. Hence, by denoting by $A$ one of the possible values of ${ }_{1} X$ and by $B$ one of the possible values of ${ }_{2} X$ it turns out to be $\mathbf{P}(A \wedge B)=\mathbf{P}(A) \mathbf{P}(B \mid A)=\mathbf{P}(B) \mathbf{P}(A \mid B)$, with $A \wedge B=B \wedge A$, as regards each covariant component of $p$ ([17], [18], [21]). I denoted by $A \wedge B=B \wedge A$ the logical product of two events while I considered $\mathbf{P}(A \wedge B)$ as a probability of their joint. In general, from the notion of conditional probability denoted by $\mathbf{P}(E \mid H)$ it is always possible to deduce that the notion of subjective probability is relative to the current state of information of a given individual represented by $H$. This operationally means that $\mathbf{P}(E \mid H)$ is the price to be paid for a conditional bet which is annulled if $H$ does not occur. Conversely, this conditional bet is won if $H$ and $E$ occur while it is lost if $H$ occurs and $E$ does not occur. I evidently considered a tri-event denoted by $E \mid H$ with values $1|1=1,0| 1=0$, $0|0=1| 0=\emptyset$. It represents only a formal variation with respect to the starting delimitation because $\emptyset=$ void is added to the two starting values $1=$ true and $0=$ false. Any tri-event can always be expressed by means of two events from a conceptual point of view. This means that all tri-events are only formally meaningful. Given a transformed bivariate random quantity ${ }_{X_{12}} t \equiv\left\{{ }_{1} X t{ }_{2} X t\right\}$, its coherent prevision $\mathbf{P}\left(x_{12} t\right)$ is again an $\alpha$-product whose metric properties remain unchanged by extending them to $\mathbf{P}$. In particular, when it turns out to be $p_{i_{1} i_{2}}=p_{i_{1}} p_{i_{2}}, \forall i_{1}, i_{2} \in I_{m}$, with $I_{m} \equiv\{1,2, \ldots, m\}$, one observes that a stochastic independence exists. Hence, one obtains $\mathbf{P}\left(x_{12} t\right)=0$, that is to say, the vectors ${ }_{(1)} \mathbf{t}$ and ${ }_{(2)} \mathbf{t}$ are $\alpha$-orthogonal. One equivalently says that the covariance of ${ }_{1} X$ and ${ }_{2} X$ is equal to 0 . 


\section{Rewriting of some fundamental metric expressions}

Now, it is possible to rewrite some fundamental metric expressions by using properly the notion of coherent prevision of bivariate random quantities that I introduced. Therefore, when one rewrites (27) and (28) it is possible to obtain

$$
{ }_{12} f \odot_{34} f=\left|\begin{array}{ll}
\mathbf{P}\left({ }_{1} X_{3} X\right) & \mathbf{P}\left({ }_{1} X_{4} X\right) \\
\mathbf{P}\left({ }_{2} X_{3} X\right) & \mathbf{P}\left({ }_{2} X_{4} X\right)
\end{array}\right|
$$

and

$$
\left\|_{12} f\right\|_{\alpha}^{2}=\left|\begin{array}{ll}
\mathbf{P}\left({ }_{1} X_{1} X\right) & \mathbf{P}\left({ }_{1} X_{2} X\right) \\
\mathbf{P}\left({ }_{2} X_{1} X\right) & \mathbf{P}\left({ }_{2} X_{2} X\right)
\end{array}\right| .
$$

On the other hand, it is known that any vector viewed as an element of a given vector space can always be expressed as a linear combination of the vectors representing a basis of the vector space under consideration. Hence, each linear combination is a division of a vector into those vectors representing a basis of the vector space under consideration. An analogous thing goes by considering (42) as well as (43), where one observes that coherent previsions of separate bivariate random quantities are basic elements of the metric expressions under consideration. I evidently accept into the domain of subjective probability a very meaningful principle borrowed from geometry according to which it is possible to divide a more complicated mathematical object into simpler mathematical objects represented by coherent previsions of bivariate random quantities. Thus, it is possible to realize that a new and fruitful notion of coherent prevision of a generic bivariate random quantity is introduced. Moreover, it is also useful when one jointly considers more than two random quantities. Indeed, one will be able to realize in due time that the notion of coherent prevision of a generic bivariate random quantity is always at the base of fundamental metric expressions related to multiple random quantities when one deals with quadratic expressions as needful metric expressions. A very important point must be stressed: the notion of coherent prevision of a univariate or bivariate random quantity is an indirect mathematical notion because its foundation is the notion of prevision of the same random quantity which is always a psychological notion in the first instance. The same thing obviously goes by considering the notion of probability of a single event. I show an approach which does not introduce arbitrary mathematical conventions but it makes more evident the distinction between an extralogical or psychological notion and a logic or mathematical notion which is nevertheless intrinsically connected to the former. According to such mathematical conventions it would be possible to give a uniquely determined answer to an indeterminate problem because of its data which are only able to establish certain limits or boundaries. These conventions must not be accepted for this reason.

\section{Conclusions}

I began to elaborate a geometric, original and extensive theory of multiple random quantities by accepting the principles of the theory of concordance into the domain of subjective probability. This acceptance is well-founded because the definition of concordance shown by Gini is implicit as well as the one of prevision of a random quantity and in particular of probability of an event. Indeed, these definitions are based on criteria which permit to measure them. I represented a generic bivariate random quantity in a tensorial fashion. I observed that metric properties of the notion of $\alpha$-product mathematically fulfill the ones of a coherent prevision of a bivariate random quantity. 
This geometric approach that I shown is useful because I am able to examine in a more complete fashion the domain of the possible alternatives by extending the notion of $\alpha$ product to two different antisymmetric tensors. Indeed, with regard to any problem that one has to consider, there always exists an enormous number of possible alternatives. If information and knowledge of a given individual do not permit to exclude some of them as impossible then all alternatives which can logically be considered remain possible for him in the sense that they are not either certainly true or certainly false. In particular, this means that he is able to consider different bivariate random quantities in addition to the starting one. This tensorial approach allows of representing a bivariate random quantity regardless of any ordered pair of univariate random quantities which are the components of the bivariate random quantity under consideration. The number of the components of an antisymmetric tensor of order 2 decreases by passing from an affine tensor of order 2 to an antisymmetric tensor of the same order and this is useful in order to satisfy simplification and compression needs. I introduced fundamental metric expressions referring to transformed random quantities representing changes of origin obtained by using a conceptually and operationally complete notion of coherent prevision of univariate random quantities. The notion of $\alpha$-norm obtained from the notion of $\alpha$-product allowed to connect vector spaces having a Euclidean structure with random quantities.

\section{References}

[1] Coletti, G., Scozzafava, R.: Probabilistic logic in a coherent setting. Kluwer Academic Publishers, Dordrecht/Boston/London (2002)

[2] de Finetti, B.: Theory of probability. J. Wiley \& Sons, 2 vol., London-New YorkSydney-Toronto (1975)

[3] de Finetti, B.: Probability, Induction and Statistics (The art of guessing). J. Wiley $\&$ Sons, London-New York-Sydney-Toronto (1972)

[4] de Finetti, B.: Probability: beware of falsifications!. Studies in subjective probability. Edited by H. E. Kyburg, jr., H. E. Smokler. R. E. Krieger Publishing Company, Huntington, New York, 195-224 (1980)

[5] de Finetti, B.: The role of "Dutch Books" and of "proper scoring rules". The British Journal of Psychology of Sciences 32, 55-56 (1981)

[6] de Finetti, B.: Probability: the different views and terminologies in a critical analysis. Logic, Methodology and Philosophy of Science VI (Hannover, 1979), 391-394 (1982)

[7] Good, I. J.: Subjective probability as the measure of a non-measureable set. Logic, Methodology and Philosophy of Science Proc. 1960 Internat. Congr., 319$329(1962)$

[8] Jeffreys, H.: Theory of probability, 3rd edn. Clarendon Press, Oxford (1961)

[9] Koopman, B. O.: The axioms and algebra of intuitive probability. Annals of Mathematics 41, 269-292 (1940)

[10] Kyburg jr., H. E., Smokler, H. E.: Studies in subjective probability. J. Wiley \& Sons, New York, London, Sydney (1964) 
[11] Pompilj, G.: On intrinsic independence. Bulletin of the International Statistical Institute, 35, 2, 91-97 (1957)

[12] Ramsey, F. P.: The foundations of mathematics and other logical essays. Edited by R. B. Braithwaite with a preface by G. E. Moore. Littlefield, Adams \& Co, Paterson, N. J. (1960)

[13] Savage, L. J.: The foundations of statistics. J. Wiley \& Sons, New York (1954)

[14] de Finetti, B.: The proper approach to probability. Exchangeability in Probability and Statistics. Edited by G. Koch, F. Spizzichino, North-Holland Publishing Company, Amsterdam, 1-6 (1982)

[15] de Finetti, B.: Probability and statistics in relation to induction, from various points of view. Induction and statistics, CIME Summer Schools, Springer, Heidelberg 18, 1-122 (2011)

[16] de Finetti, B.: Probabilism: A Critical Essay on the Theory of Probability and on the Value of Science. Erkenntnis 31, 2/3, 169-223 (1989)

[17] Coletti, G., Petturiti, D., Vantaggi, B.: When upper conditional probabilities are conditional possibility measures. Fuzzy Sets and Systems 304, 45-64 (2016)

[18] Coletti, G., Petturiti, D., Vantaggi, B.: Conditional belief functions as lower envelopes of conditional probabilities in a finite setting. Information Sciences 339, 64-84 (2016)

[19] Coletti, G., Scozzafava, R., Vantaggi, B.: Possibilistic and probabilistic logic under coherence: default reasoning and System P. Mathematica Slovaca 65, 4, $863-890(2015)$

[20] Coletti, G., Petturiti, D., Vantaggi, B.: Bayesian inference: the role of coherence to deal with a prior belief function. Statistical Methods \& Applications, 23, 4, $519-545$ (2014)

[21] Gilio, A., Sanfilippo, G.: Conditional random quantities and compounds of conditionals. Studia logica, 102, 4, 709-729 (2014)

[22] McCullagh, P.: Tensor methods in statistics. Chapman and Hall, London-New York (1987)

[23] Pistone, G., Riccomagno, E., Wynn, H. P.: Algebraic statistics. Chapman \& Hall, Boca Raton-London-New York-Washington, D.C. (2001)

[24] Gini, C.: Statistical methods. Istituto di statistica e ricerca sociale "Corrado Gini". Università degli studi di Roma, Roma (1966)

[25] Gini, C.: The contributions of Italy to modern statistical methods. Journal of the Royal Statistical Society, 89, 4, 703-724 (1926) 